Methods Data on all CD patients, including those with persisting symptoms and tertiary referrals, was collected prospectively from 1998-2018. Patients were systematically investigated to establish the aetiology of their continued symptoms. They were also referred to a specialist coeliac dietitian to identify any lapses in GFD adherence or gluten cross-contamination. A repeat duodenal biopsy was performed and compared to previous biopsies where possible to check for histological remission. Colonoscopy, lactose hydrogen breath test, glucose hydrogen breath test, SeHCAT scan, CLO testing, faecal elastase, immunohistochemistry and $\gamma$-TCR clonality were performed.

Results 2,356 patients with suspected CD were seen in this time period (121 were tertiary referrals). 157 were excluded from analysis due to unconfirmed diagnosis. Of the remaining 2,199 patients with confirmed CD, 2,123 had both villous atrophy and positive IgA-EMA/TTG, and 76 had seronegative CD. Of the 2,199 patients with CD (67\% female, mean age at diagnosis $42.8 \pm 18.5), 292(13 \%)$ had persisting symptoms. The leading causes for persisting symptoms in patients without RCD $(73 \%$ female, mean age at diagnosis $35.7 \pm$ 19.2) were: gluten contamination (22\%), functional/irritable bowel syndrome (20\%), pancreatic exocrine insufficiency $(7 \%)$, reflux dysmotility (5\%), and microscopic colitis (5\%). Of a total of 74 patients who were identified with RCD, 56 had RCD I (71\% female, mean age at CD diagnosis $41.8 \pm 19.0)$ and 18 had RCD II $(33 \%$ female, mean age at CD diagnosis $55.4 \pm 13.3$ ). After a median follow up of 40.5 months (IQR 21.8-73.3), mortality was 7\% in the RCD I group, compared to $39 \%$ in the RCD II group $(\mathrm{p}=0.019)$. Higher age at diagnosis of $\mathrm{CD}$ is a predictor for having RCD in patients with persisting symptoms $(\mathrm{p}<0.001)$.

Conclusions This is the largest UK study of NRCD and RCD. The contemporary mortality data in RCD II remains poor. Patients with suspected RCD should be referred to the National Centre for consideration of novel therapies such as IL-15 and Stem Cell Transplant.

\section{OWE-21 SERONEGATIVE VILLOUS ATROPHY OF UNKNOWN ORIGIN DISPLAYS DISTINCTIVE CLINICAL AND GENETIC FEATURES AND NATURAL HISTORY}

\footnotetext{
1,2Annalisa Schiepatti ${ }^{*},{ }^{1}$ David S Sanders, ${ }^{3}$ John Goodwin, ${ }^{3}$ Tim Key, ${ }^{2}$ Paolo Giuffrida, ${ }^{4}$ Annalisa De Silvestri, ${ }^{2}$ Federico Biagi. ${ }^{1}$ Academic Unit of Gastroenterology, Royal Hallamshire Hospital, Sheffield, UK, Sheffield, UK; ${ }^{2}$ Coeliac Centre, First Department of Internal Medicine, University of Pavia, Pavia, Italy; ${ }^{3} \mathrm{NHS}$ Blood and Transplant, UK; ${ }^{4}$ Clinical Epidemiology, IRCCS Policlinico San Matteo, Pavia, Italy
}

10.1136/gutjnl-2019-BSGAbstracts.309

Introduction Seronegative villous atrophies of unknown origin (SNVA-UO) are rare and poorly defined. Aims: i) To classify SNVA-UO and study their clinical features, histology, HLA and natural history. ii) To compare genetics and survival between SNVA-UO and patients affected by coeliac disease (CD) and complicated CD.

Methods Notes of SNVA-UO patients from two referral centres were retrospectively reviewed and data on follow-up prospectively collected until 01/2019. CD and its complications and other causes of SNVA (autoimmune enteropathy, common variable immunodeficiency, olmesartan enteropathy, parasites, etc) were thoroughly excluded. Evidence of lymphoproliferative features (TCR- $\gamma$ clonality on duodenal biopsies and/or past history of extra-intestinal lymphoproliferative disorders) and persistent villous atrophy (VA) during follow-up were used as criteria to classify them, as follows. GROUP 1: SNVA without lymphoproliferative features and with spontaneous recovery of VA. GROUP 2: persistent SNVA without lymphoproliferative features. GROUP 3: persistent SNVA with lymphoproliferative features. HLA DQA1 and DQB1 allelic frequencies were compared using $\chi 2$ test and Fisher test. Survival was analysed by means of Kaplan-Meier curves.

Results 76 patients with SNVA-UO were enrolled. 50 were included in group 1 (26F, age at diagnosis $49 \pm 18$ years), 14 in group $2(7 \mathrm{~F}, 43 \pm 14), 12$ in group $3(5 \mathrm{~F}, 52 \pm 17)$. VA spontaneously normalized in 47 patients in group 1 after a median of 10 months, IQR 5-14.5. Histological response occurred in 4 patients in group 2 on traditional immunosuppressants. Survival analysis showed significant differences $(\mathrm{p}<0.001)$, with group 2 characterised by long-term survival (100\% alive, median follow-up 61 months, IQR 50.5-97.5) and group 3 by the poorest prognosis and a mortality higher than complicated CD $(58 \%$ of patients dead after a median of 11 months from diagnosis, IQR 9.5-40). Group 1 displayed a favourable outcome similar to $\mathrm{CD}$. Group 2 shows a high frequency of HLA-DQB1*0301 and DQB1*06, while HLA$\mathrm{DQB} 1 * 02$ was more frequent in group 3 and in group 1. Partial VA, dyspepsia and absence of weight loss predicted inclusion into group $1 \quad(\mathrm{p}<0.001)$, while hypoalbuminemia $(<3.5$ $\mathrm{g} / \mathrm{dL})$ inclusion into group $3(\mathrm{p}<0.02)$.

Conclusions For the first time we demonstrated that SNVAUO is made by heterogeneous enteropathies with distinct clinical features, genetics and prognosis. Clinical management should be tailored accordingly.

\section{Posters}

\section{PWE-022 THE INCIDENCE OF COELIAC DISEASE IN PATIENTS PRESENTING WITH STRESS FRACTURE TO A TERTIARY CENTRE}

${ }^{1} \mathrm{R}$ Smith, ${ }^{1} \mathrm{~J}$ Baldock, ${ }^{2} \mathrm{M}$ FitzPatrick* ${ }^{1} \mathrm{~N}$ Jones, ${ }^{1} \mathrm{~J}$ Newton. ${ }^{1}$ Department of Sport and Exercise Medicine, Nuffield Orthopaedic Centre, Oxford University Hospitals NHS Foundation Trust, Oxford; ${ }^{2}$ Translational Gastroenterology Unit, University of Oxford, Oxford

\subsection{6/gutjil-2019-BSGAbstracts.310}

Introduction Stress fractures, fatigue-induced bone fractures caused by repetitive mechanical stress, are often multifactorial in nature and are associated with a number of metabolic bone disorders. Whilst the association between coeliac disease (CD) and osteoporosis is well-recognised, it is unclear whether an association between $\mathrm{CD}$ and stress fractures exists, with only rare case reports in the literature. This study aimed to examine the incidence of $\mathrm{CD}$ in a prospective cohort of patients presenting with stress fractures to a specialist NHS Sport and Exercise Medicine (SEM) clinic.

Methods An analysis of a prospective cohort of 100 consecutive patients with radiologically-proven stress fractures who presented to a single tertiary UK SEM clinic was performed. Electronic health records were used to examine fracture site, comorbidities, tissue transglutaminase (TTG) result, biochemistry, haematinics, dual energy x-ray absorptiometry, and endoscopy findings.

Results Seventy patients in the cohort were female (70\%) and the mean age was 37 years (range 18-69). Two patients had pre-existing coeliac disease $(2 \%)$. Coeliac serology was performed for 85 patients (85\%), with 5 additional patients 